\title{
Parton Distribution Functions and Lattice QCD
}

\author{
Huey-Wen $\operatorname{Lin}^{1,2, \star}$ \\ ${ }^{1}$ Department of Physics and Astronomy, Michigan State University, East Lansing, Ml 48824 \\ ${ }^{2}$ Department of Computational Mathematics, Michigan State University, East Lansing, MI 48824
}

\begin{abstract}
Recently, there have been rapid developments in lattice-QCD calculations of proton structure, especially in the parton distribution functions (PDFs). We overcame a longstanding obstacle and for the first time in lattice-QCD are able to directly calculate the Bjorken- $x$ dependence of the quark, helicity and transversity distributions. The PDFs are obtained using the large-momentum effective field theory (LaMET) framework where the full Bjorken- $x$ dependence of finite-momentum PDFs, called "quasi-PDFs", can be calculated on the lattice. The quasi-PDF nucleon matrix elements are renormalized nonperturbatively in RI/MOM-scheme. Following a nonperturbative renormalization of the parton quasi-distribution in a regularization-independent momentum-subtraction scheme, we establish its matching to the $\overline{\mathrm{MS}} \mathrm{PDF}$ and calculate the non-singlet matching coefficient at next-to-leading order in perturbation theory. In this proceeding, I will show the progress that has been made in recent years, highlighting the latest state-of-the art PDF calculations at the physical pion mass. Future impacts on the large- $x$ global PDF fits are also discussed.
\end{abstract}

\section{Introduction}

Parton distribution functions (PDFs) are important quantities describing the probability densities of quarks and gluons within hadrons. They are not only fundamental properties of quantum chromodynamics (QCD) but also are key inputs to predict cross sections in high-energy scattering experiments and to aid new-physics search large hadron collider. Calculating the $x$-dependence of PDFs from first principles has long been a holy grail for nuclear and high-energy physics. In modern parton physics, the PDFs are defined from the lightcone correlations of quarks and gluons in the hadron, so they involve strong infrared dynamics and can only be solved by nonperturbative methods such as lattice QCD. However, the direct calculation of PDFs on a Euclidean lattice has been extremely difficult because the real-time dependence of the lightcone makes it infeasible to extract them from lattice simulations with imaginary time. Early lattice-QCD studies based on the operator product expansion (OPE) could only access the lower moments of the PDF.A similar situation also occurs in lattice calculations of other parton observables, such as the distribution amplitudes (DAs) and generalized parton distributions (GPDs).

In recent years, there has been a promising proposal, large-momentum effective theory (LaMET), [1] wherein the PDFs and other parton observables can be directly extracted from the lattice by calculating the matrix elements of certain static operators in a boosted hadron state. For the

^e-mail: hwlin@pa.msu.edu 
unpolarized quark PDF, the static operator is $O_{\Gamma}(z)=\bar{\psi}(z) \Gamma U(z, 0) \psi(0)$, where the spacelike Wilson line $U(z, 0)=P \exp \left(-i g \int_{0}^{z} d z^{\prime} A^{z}\left(z^{\prime}\right)\right)$ so that under the infinite Lorentz boost along the $z$-axis, $O_{\Gamma}$ approaches the lightcone correlation operator that defines the PDF. The hadron matrix element of $O_{\Gamma}(z)$ can be directly obtained from lattice QCD, and its Fourier transformation is known as the quasi-PDF:

$$
\tilde{q}\left(x, P_{z}, \tilde{\mu}\right)=\int_{-\infty}^{\infty} \frac{d z}{2 \pi} e^{i x P_{z} z}\left\langle P\left|O_{\Gamma}(z)\right| P\right\rangle .
$$

The quasi-PDF is related to the lightcone PDF through a factorization theorem, where the former can be factorized into a perturbative matching coefficient and the latter, up to power corrections suppressed by the nucleon momentum. This factorization theorem is founded in LaMET [1-5], where the matching coefficient can be calculated exactly in perturbation theory.

Since the proposal of LaMET, there has been much progress; on the theoretical side, there are the matching coefficients connecting the quasi-PDFs to the PDFs at one-loop orderthe nucleon-mass correction, and the renormalization properties of the quasi-PDF.There has also been progress on latticeQCD simulation, such as the isovector quark PDF of the nucleon [6-10], the meson DAs [11, 12] and the nonperturbative renormalization in the regularization-independent momentum subtraction (RI/MOM) scheme $[13,14]$. Certain technical issues regarding the nonperturbative renormalization were raised and addressed in Refs. [10, 13-18]. In parallel, there have also been other proposals to calculate the PDFs in lattice QCD [19-27] which are subject to their own systematics; nonetheless, they can be complementary to each other as well as to the LaMET approach.

In this proceeding, I report on state-of-the-art lattice calculations of the unpolarized, longitudinally and transversely polarized isovector quark distributions at physical pion mass using the LaMET method. The quasi-PDFs with nucleon momenta as large as $3.0 \mathrm{GeV}$ are calculated and multiple source-sink separations are used to remove excited-state contamination. The nucleon matrix elements are renormalized using RI/MOM scheme, and a corresponding matching calculation is used to connect the RI/MOM quasi-PDF to the $\overline{\mathrm{MS}}$ renormalized lightcone PDF. Our result shows a significant improvement compared to previous lattice studies, in particular in the moderate to large- $x$ region. It also signals a promising trend that the precision of lattice calculations is approaching the precision of phenomenological studies with increasing computing resources.

\section{Parton Distribution Functions at Physical Pion Mass}

\subsection{LaMET}

On the lattice one computes a time-independent spatially displaced matrix element that can be connected to the PDF. A convenient choice for leading-twist PDFs is to take the hadron momentum and quark-antiquark separation to be along the $z$ direction

$$
h_{\Gamma}\left(z, p_{z}\right)=\frac{1}{4 p_{z}} \sum_{s=1}^{2}\left\langle p, s\left|\bar{\psi}(z) \Gamma e^{i g \int_{0}^{z} A_{z}\left(z^{\prime}\right) d z^{\prime}} \psi(0)\right| p, s\right\rangle,
$$

where $p_{z}$ is the hadron momentum boosted in the $z$ direction, $s$ its spin, and $z$ is the separation of the quark and antiquark fields $\bar{\psi}$ and $\psi$. There are multiple choices of operator in this framework that will recover the same lightcone PDFs when the large-momentum limit is taken. For example, $\Gamma$ can be $\gamma_{z}$ or $\gamma_{t}$ [21, 28-30]; both will give the unpolarized PDFs in the infinite-momentum frame.

With the matrix elements obtained on the lattice, one can calculate a "quasi-PDF" by

$$
\widetilde{f}\left(x, 1 / a, p_{z}\right)=\int \frac{d z}{2 \pi} e^{-i x z p_{z}} p_{z} h_{\Gamma}\left(z, p_{z}\right)
$$


where $a$ is the lattice spacing. In the context of LaMET, this can be related to PDFs using the matching condition

$$
\widetilde{q}\left(x, \Lambda, p_{z}\right)=\int_{-1}^{1} \frac{d y}{|y|} Z\left(\frac{x}{y}, \frac{\mu}{p_{z}}, \frac{\Lambda}{p_{z}}\right)_{\mu^{2}=Q^{2}} q\left(y, Q^{2}\right)+O\left(\frac{\Lambda_{\mathrm{QCD}}^{2}}{p_{z}^{2}}, \frac{M^{2}}{p_{z}^{2}}\right),
$$

where $\mu$ is the renormalization scale, $Z$ is a matching kernel and $M$ is the hadron mass. Here the $O\left(M^{2} / p_{z}^{2}\right)$ terms are target-mass corrections and the $O\left(\Lambda_{\mathrm{QCD}}^{2} / p_{z}^{2}\right)$ terms are higher-twist effects, both of which are suppressed at large hadron momentum. Early exploratory works have shown great promise in obtaining quantitative results for the unpolarized, helicity and transversity quark and antiquark distributions $[6,8,31,32]$.

\subsection{Lattice Details and Procedure}

We perform lattice calculations of the bare isovector quark unpolarized, helicity, and transversity quasi-PDF using clover valence fermions on an ensemble of 884 gauge configurations with lattice spacing $a=0.09 \mathrm{fm}$, box size $L \approx 5.8 \mathrm{fm}$, and with pion mass $M_{\pi} \approx 135 \mathrm{MeV}$ and $N_{f}=2+1+1$ (degenerate up/down, strange and charm) flavors of highly improved staggered dynamical quarks (HISQ) [33] generated by MILC Collaboration [34]. The gauge links are one-step hypercubic (HYP)smeared [35] to suppress discretization effects. The clover parameters are tuned to recover the lowest pion mass of the staggered quarks [36-39]. We use multigrid algorithm [40, 41] in Chroma software package [42] to speed up the clover fermion inversion of the quark propagator at physical pion mass, allowing a high-statistics calculation.

We use Gaussian momentum smearing [43] for the quark field $\psi(x)+\alpha \sum_{j} U_{j}(x) e^{i k \hat{e}_{j}} \psi\left(x+\hat{e}_{j}\right)$, where $k=6$ is the input momentum parameter, $U_{j}(x)$ are the gauge links in the $j$ direction, and $\alpha$ is a tunable parameter as in traditional Gaussian smearing. Such a momentum smearing is designed to increase overlap of the lattice sources with the ground-state proton of the desired momenta, which allows us to reach higher-momentum states than was previously possible [10]. This calculation employs sources with $\vec{P}=\left\{0,0, n \frac{2 \pi}{L}\right\}$, with $n \in\{10,12,14\}$, which correspond to $2.2,2.6$ and $3.0 \mathrm{GeV}$ proton momenta, respectively.

We first calculate the equal-time three-point correlator along the $z$-axis with operator $\hat{O}(z, a)=$ $\bar{\psi}_{q}(z) i \Gamma U(z, 0) \psi_{q}(0)$ with the Wilson line $U(z, 0)=P \exp \left(-i g \int_{0}^{z} d z^{\prime} A_{z}\left(z^{\prime}\right)\right)$ and subscript $q$ as a flavor index. We calculate the flavor combination $\delta \widetilde{u}-\delta \widetilde{d}$ so that the disconnected diagrams cancel on the lattice. For the nucleon matrix elements of $\hat{O}(z, a)$ at a given boost momentum, $\widetilde{h}\left(z, P_{z}, a\right)$, we calculate six source-sink separations $t_{\text {sep }} \in\{0.54,0.72,0.81,0.90,0.99,1.08\} \mathrm{fm}$, with $\{16,32,32,64,64,64\}$ thousand measurements among 884 gauge configurations, respectively. Following the work in Ref. [39], each three-point correlator, $C_{\Gamma}^{(3 \mathrm{pt})}\left(P_{z}, t, t_{\mathrm{sep}}\right)$ can be decomposed as

$$
\begin{aligned}
C_{\Gamma}^{3 \mathrm{pt}}\left(P_{z}, t, t_{\mathrm{sep}}\right) & =\left|\mathcal{A}_{0}\right|^{2}\left\langle 0\left|O_{\Gamma}\right| 0\right\rangle e^{-E_{0} t_{\mathrm{sep}}} \\
& +\left|\mathcal{A}_{1}\right|^{2}\left\langle 1\left|O_{\Gamma}\right| 1\right\rangle e^{-E_{1} t_{\mathrm{sep}}} \\
& +\mathcal{A}_{1} \mathcal{A}_{0}^{*}\left\langle 1\left|O_{\Gamma}\right| 0\right\rangle e^{-E_{1}\left(t_{\mathrm{sep}}-t\right)} e^{-E_{0} t} \\
& +\mathcal{A}_{0} \mathcal{A}_{1}^{*}\left\langle 0\left|O_{\Gamma}\right| 1\right\rangle e^{-E_{0}\left(t_{\mathrm{sep}}-t\right)} e^{-E_{1} t}+\ldots
\end{aligned}
$$

where the source point has been shifted to zero for each measurement, the operator is inserted at time $t$, and the nucleon state is annihilated at the sink time $t_{\text {sep }}$, which (after shifting) is also the sourcesink separation. The state $|0\rangle$ represents the ground state and $|n\rangle$ with $n>0$ the excited states. In our two-state fits, the amplitudes $\mathcal{A}_{i}$ and the energies $E_{i}$ are functions of $P_{z}$ and can be obtained from the corresponding two-point correlators. Here we investigate the excited-state contamination 
by performing fits with and without the $\left\langle 1\left|O_{\Gamma}\right| 1\right\rangle$ contribution (and labeled them as "two-simRR" and "two-sim" methods, respectively) and using different inputs of source-sink separations $t_{\text {sep }}$ shown in Refs. [44-46]. The two-simRR analysis using $t_{\text {sep }}$ as small as $0.54 \mathrm{fm}$ gives consistent results with the two-sim analysis using $t_{\text {sep }}=0.81 \mathrm{fm}$, with approximately the same statistical errors after removing the excited-state contamination. Similar results are given by two other fits with larger error as they use fewer three-point proton correlators. Our final result uses the "two-simRR" fit with three-point correlators data of $t_{\text {sep }}$ ranging from 0.72 to $1.08 \mathrm{fm}$.

As a second step, we calculate the nonperturbative renormalization (NPR) factor $\widetilde{Z}\left(z, p_{z}^{R}, \mu_{R}, a\right)$ from the amputated Green function of $\hat{O}(z, a)$ with a similar procedure to that defined in Ref. [46, 47], where $p_{z}^{R}$ and $\mu_{R}$ are the Euclidean quark momentum in the $z$-direction and the off-shell quark momentum, respectively. The bare matrix element of $\hat{O}(z, a), \widetilde{h}\left(z, P_{z}, a\right)$, has ultraviolet (UV) power and logarithmic divergences as $a \rightarrow 0$ and must be nonperturbatively renormalized to have a well defined continuum limit.

The NPR factor $\tilde{Z}\left(z, p_{z}^{R}, \mu_{R}, a\right)$ is calculated using the off-shell quark matrix element of $\hat{O}(z, a)$ and requiring that all the loop corrections are canceled by $\tilde{Z}\left(z, p_{z}^{R}, \mu_{R}, a\right)$ at given $p_{z}^{R}$ and $\mu_{R}$. It is computed in Landau gauge using the same lattice ensemble to compute $\widetilde{h}$. The renormalized matrix element $\widetilde{h}_{R}\left(z, P_{z}, p_{z}^{R}, \mu_{R}\right)=\widetilde{Z}^{-1}\left(z, p_{z}^{R}, \mu_{R}, a\right) \widetilde{h}\left(z, P_{z}, a\right)$ inherits the dependence on $p_{z}^{R}$ and $\mu_{R}$, which is supposed to be canceled after the later matching step. However, since our matching coefficient is only available at one-loop order, there will be remnant dependence on $p_{z}^{R}$ and $\mu_{R}$ in the final $\delta q(x, \mu)$. On the other hand, the lattice discretization effects of order $O\left(a p_{z}^{R}, a \mu_{R}\right)$ or higher are also expected, since we do not take the continuum limit. Both factors will lead to systematic uncertainties in our analysis, so we estimate them by varying the values of $p_{z}^{R}$ from 1.3 to $3 \mathrm{GeV}$, and $\mu_{R}$ between 2.3 and $3.7 \mathrm{GeV}$. Our results show an insensitivity to $\mu_{R}$ but noticeable dependence on $p_{z}^{R}$ for all three PDFs [44-46]. We choose $\widetilde{h}_{R}\left(z, P_{z}, p_{z}^{R}, \mu_{R}\right)$ at $\mu_{R}=3.7 \mathrm{GeV}$ and $p_{z}^{R}=2.2 \mathrm{GeV}$ to be the central value; we include the variation of $\mu_{R}$ and $p_{z}^{R}$ as sources of systematic uncertainties.

Next, we Fourier transform the $\widetilde{h}_{R}\left(z, P_{z}, p_{z}^{R}, \mu_{R}\right)$ into $x$-space to obtain the quasi-distribution $\delta \widetilde{q}\left(x, P_{z}, p_{z}^{R}, \mu_{R}\right)$. As shown in Refs. [44-46], the long-range correlation, which dominates the small$x$ distribution, has much larger statistical uncertainty, and the higher-twist effects as well as finitevolume effects will also become important with larger $|z|$. Therefore, we have to truncate the Fourier transform at a finite $\left|z_{\max }\right|$, which will limit our prediction for the small- $x$ distribution and introduce an unphysical oscillation in $x$-space. This can be removed using the "derivative" method proposed in our earlier work [10]:

$$
\tilde{Q}\left(x, P_{z}, p_{z}^{R}, \mu_{R}\right)=i \int_{-z_{\max }}^{z_{\max }} d z e^{i x P_{z} z} \tilde{h}_{R}^{\prime}\left(z, P_{z}, p_{z}^{R}, \mu_{R}\right) / x,
$$

where $\tilde{Q}$ is quasi-PDFs $\left(q(x), \Delta q(x)\right.$ and $\delta q(x)$ respectively), $\tilde{h}_{R}^{\prime}$ is the derivative of the renormalized matrix elements for the corresponding operators. At the end of the day, we vary $\left|z_{\max }\right|$ to estimate the remaining corresponding error, which turns out to be small compared with other systematics.

\subsection{Unpolarized PDF}

For the unpolarized PDF, we use the $\Gamma=\gamma_{t}$ operator, which is free from mixing, and the corresponding matching derived in Ref. [44]. The matching raises the antiquark (i.e. negative- $x$ region) distribution to the same asymmetry for $x<-0.05$, and lowers the positive mid- $x$ to large- $x$ quark distribution, compared with our exploratory study and heavier-pion PDF. We study the dependence on the nucleon boost momentum, shown on the left-hand side of Fig. 1. Within the statistical errors, the distribution seems to converge across the three momenta. However, the central values shift noticeably from 2.2 
to $3.0 \mathrm{GeV}$, moving the antiquark distribution toward the asymmetry measured in experiment: $\bar{d}(x)>$ $\bar{u}(x) . L P^{3}$ 's final unpolarized PDF, shown on the right-hand side of Fig. 1, significantly improves on the previous results at physical pion mass [10]. We increase the nucleon momenta used in the calculation from $0.4,0.8,1.3 \mathrm{GeV}$ to $2.2,2.6$ and $3.0 \mathrm{GeV}$. We use $O_{\gamma_{t}}$ operator here rather than the $O_{\gamma_{z}}$ used in Ref. [13]. (Though we showed in Ref. [13] that the final PDF is consistent when including the scalar matrix elements.) We extend our matrix-element analysis to include an extra term due to excited states. We increase statistics by more than a factor of 10 . We also include the complete matching needed to convert the RI/MOM quasi-PDF into the $\overline{\mathrm{MS}}$ lightcone PDF.
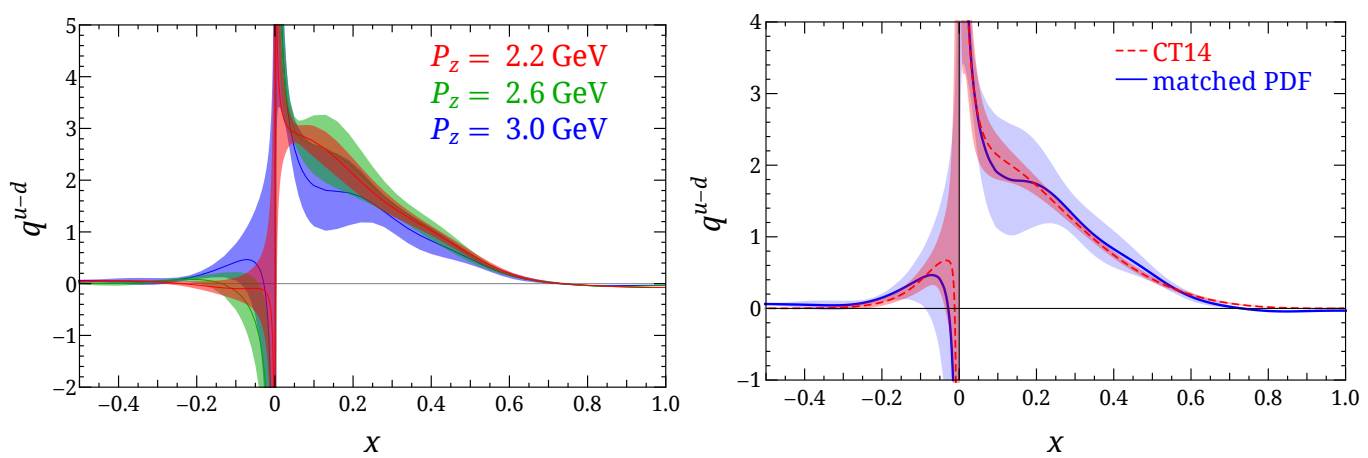

Figure 1. (Left) Nucleon boost-momentum dependence of the matched unpolarized isovector PDFs. For the quark asymmetry, the shape is consistent throughout most $x$ regions. However, in the antiquark region, there is a significant change in distribution as momentum increases. (Right) Our final PDF renormalized at $3 \mathrm{GeV}$ and compared with CT14 [48], which is consistent with NNPDF3.1 [49] and CJ15 [50]. Our results agree nicely with the global-analysis PDF. More details about these figures can be found in Ref. [44].

\subsection{Helicity}

The isovector quark helicity distribution, obtained at the largest proton momentum of $3 \mathrm{GeV}$, is shown on the left-hand side of Fig. 2. The statistical error (with the excited-state contamination subtracted based on two-state fits) is shown as the red band. The systematic uncertainty, shown combined with the statistical one as the gray band in Fig. 2, is obtained by varying the scales in the NPR for $\mu_{R} \in$ $\{2.3,3.7\} \mathrm{GeV}$ and $p_{z}^{R} \in\{1.3,3\} \mathrm{GeV}$, and includes the error from the one-loop matching inversion. Since we only have configurations with one lattice spacing and volume, the associated systematic errors are beyond our estimation. The target-mass correction from Ref. [8] is found to be negligible for all three nucleon momenta, again indicating small higher-twist contributions. Also shown in the figure are the phenomenological fits from NNPDFpol1.1 [51] and JAM [52]. The present calculation is consistent with experiment within $2 \sigma$ in the large- $x$ region. For $x$ very close to 1 , the calculation is in principle limited by the finite lattice spacing effect at large $P_{z}$, where the proton needs be resolved with a finer longitudinal scale because of Lorentz contraction. For $x<0.1$, the present calculation is limited by the accuracy of large- $z P_{z}$ data. As in experiment, determining the small- $x$ PDFs requires large-momentum hadrons.

The present calculation shows the potential impact of lattice simulations combined with the LaMET approach in determining PDFs. The JLab $12-\mathrm{GeV}$ program is well positioned to make large$x$ determinations of polarized and unpolarized parton distributions, which are extremely valuable to 
interpret large $P_{T}$ events at the Large Hadron Collider. Lattice calculations at $10 \%$ level will already be very useful in deciding the large- $x$ behavior, cross-checking with the experimental data.

\subsection{Transversity}

$L P^{3}$ 's transversity result [46] (taken from the physical transversity with $P_{z}=3.0 \mathrm{GeV}$ ) is compared with semi-elastic DIS (SIDIS) fits from JAM17 and LMPSS17 [53] on the right-hand side of Fig. 2. The error bands of $L P^{3}$ 's transversity, shown in Fig. 2, contain two types of systematics: First, using the continuum and infinite-volume extrapolations of $g_{T}$ in Ref. [54], which includes the same lattices used in this work, we conservatively estimate our lattice systematics due to $g_{T}$ used in normalization (4\%), finite volume $(0.5 \%)$, nonzero lattice spacing (enlarged from $a$ to $3 a$ to compensate for the length contraction of a moving proton) (3.5\%), which add up to $5.4 \%$. Second, the renormalizationscale dependence in the NPR procedure, which is the dominant systematic. These two types of systematics are combined in quadrature to give our final result. Our prediction for $\delta q(x, \mu)$ is consistent with the global analysis of JAM17 but with a significantly smaller uncertainty and an unequivocally positive-definite isovector distribution over the range $0<x<1$. Compared to the LMPSS17 constrained fit using lattice averaged $g_{T}$, our result shows a remarkable agreement within $2 \sigma$. Moreover, in contrast to the recent calculation by ETMC [55], our antiquark distribution favors a vanishing flavor asymmetry in the antiquark sea, which has been assumed in all global analyses so far. Based on our earlier works $[10,13,18]$, we speculate that this is due to a systematic error from the truncation in the Fourier transformation. As shown in Fig. 4 of Ref. [10], the antiquark region is highly sensitive to the proton momentum used in the calculation. In this work, our proton momentum $P_{z}=3.0 \mathrm{GeV}$ is about factor of 2 larger than that in ETMC's $(1.4 \mathrm{GeV})$, which could also explain the difference in our results.
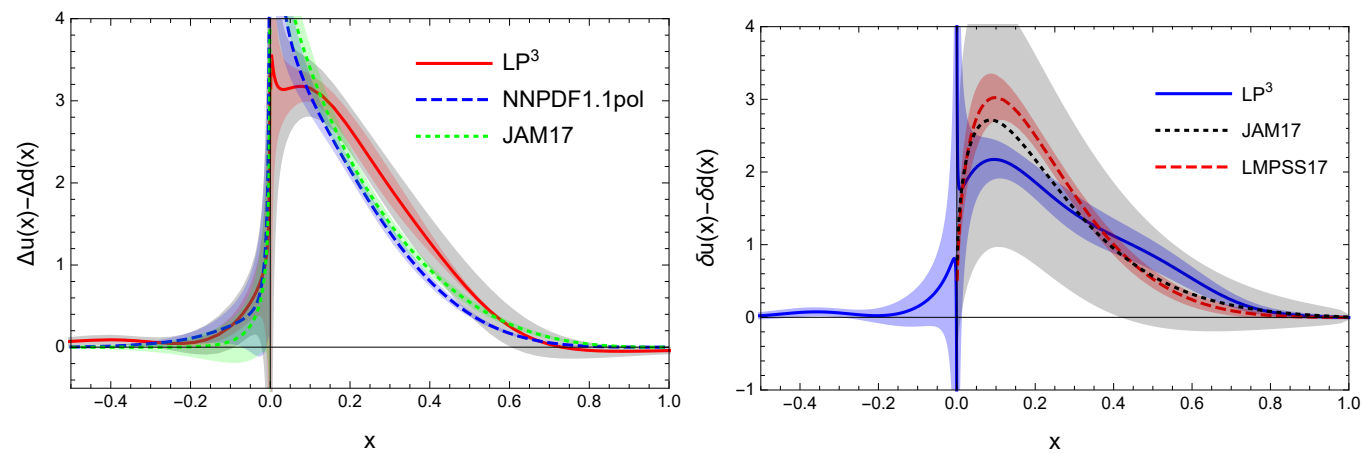

Figure 2. (Left) $L P^{3}$ 's isovector quark helicity PDF [45] matched in $\overline{\mathrm{MS}}$-scheme at scale $\mu=3 \mathrm{GeV}$, extracted from LaMET at the largest proton momentum ( $3 \mathrm{GeV})$, compared with fits by NNPDFpol1.1 [51] and JAM [52]. The red band contains statistical error, while the gray band also includes systematics. (Right) $L P^{3}$ 's proton isovector transversity PDF [46] at renormalization scale $\mu=\sqrt{2} \mathrm{GeV}$ ( $\overline{\mathrm{MS}}$ scheme), extracted from lattice QCD and LaMET at $P_{z}=3 \mathrm{GeV}$, compared with global fits by JAM17 and LMPSS17 [53]. The blue error band includes statistical errors (which fold in the excited-state uncertainty) and systematics.

\section{Lattice Constraints for Global PDFs Fits}

How can the current lattice-QCD isovector PDFs calculation contribute to the global PDF fits in the next 5 years? Currently, the large- $x$ region of the PDF is not well constrained by the experimental data 
in the global fits, or suffers hard-to-quantify nuclear-theory uncertainty. The lattice isovector PDFs at large- $x$ region, does not have large boost-momentum dependence (unlike the small- $x$ region), so it should be able to provide good constraints to the global PDF fit.

The whitepaper [56] from the first joint-community workshop between the LQCD and global analysis have gives an estimation of the precision needed and its corresponding impacts on the global PDF fits for the most-studied unpolarized PDF and helicity cases.

Firstly, a set of pseudo-data for the isovector combinations at $Q^{2}=4 \mathrm{GeV}^{2}$

$$
u\left(x_{i}, Q^{2}\right)-d\left(x_{i}, Q^{2}\right) \quad \text { and } \quad \bar{u}\left(x_{i}, Q^{2}\right)-\bar{d}\left(x_{i}, Q^{2}\right), \quad i=1, \ldots, N_{x},
$$

for the unpolarized case, and for

$$
\Delta u\left(x_{i}, Q^{2}\right)-\Delta d\left(x_{i}, Q^{2}\right) \quad \text { and } \quad \Delta \bar{u}\left(x_{i}, Q^{2}\right)-\Delta \bar{d}\left(x_{i}, Q^{2}\right), \quad i=1, \ldots, N_{x},
$$

for the polarized case, with $N_{x}$ being the number of points in $x$-space that are being sampled. For this study, we choose the $N_{x}=5$ points to be

$$
x_{i}=0.70,0.75,0.80,0.85,0.90 .
$$

for lattice-QCD computations.

Three scenarios for the total uncertainty of $\delta_{L}^{(i)}=12 \%, 6 \%$ and $3 \%$ are denoted by scenario D, E, and F, respectively. For each scenario, we assume the same relative error for each value of $\left\{x_{i}\right\}$, and we neglect possible correlations between neighboring $x$-points.

We summarize the results of this exercise in Fig. 3, where we plot the ratio of the PDF uncertainties in each scenario D, E and F to the uncertainty of the original NNPDF3.1 (NNPDFpol1.1) set. We show the impact on the PDF uncertainties in $\bar{u}$ and $\bar{d}$ at large- $x$ in the upper plots, with the corresponding comparison for $\Delta \bar{u}$ and $\Delta \bar{d}$ in the lower plots. We concentrate on the results for the individual quark flavors, even though the constraints are imposed on differences between flavors, since the former are of the more direct interest for phenomenology.

From this comparison, we find that lattice-QCD calculations of the $x$-dependence of PDFs can significantly reduce the uncertainties for both unpolarized and polarized antiquarks in the large- $x$ region. Taking into account that the PDF uncertainties on the large- $x$ antiquarks are rather large, and that they enter a number of important beyond-the-Standard Model (BSM) search channels (such as, for instance, production of new heavy gauge bosons $W^{\prime}$ and $Z^{\prime}$ ), our analysis demonstrates that such calculations would have direct phenomenological implications. In a Monte-Carlo approach such as NNPDF, the PDF uncertainties themselves fluctuate, particularly at low scales, explaining the wiggles in these plots.

Fig. 3 shows that in the unpolarized case, the large- $x$ PDF uncertainties could be reduced to $60 \%$ of their original value. We also find that there are no large differences between the three scenarios, probably because the constraint is on quark differences rather than on individual flavors, so there is freedom for $\bar{u}$ and $\bar{d}$ to vary in a correlated fashion while still satisfying the constraint. However, it does suggest that a direct lattice-QCD calculation of $x \bar{u}-x \bar{d}$ does not need to reach uncertainties at the few-percent level to influence global fits. For the polarized PDFs, Fig. 3 demonstrates that the reduction in PDF uncertainties could be significantly more marked. For instance, in the case of $\Delta \bar{d}$, at $x \simeq 0.8$ the resulting PDF uncertainty from scenario $\mathrm{F}$ is less than $50 \%$ of the original uncertainty.

\section{Conclusion and Outlook}

We have presented latest lattice-QCD results for the isovector (that is, the up-down quark asymmetry in the proton), unpolarized, helicity and transversity PDFs which have much potential impact on PDF 
$\delta(\bar{u}) @ Q^{2}=4 \mathrm{GeV}^{2}$, NNPDF3.1

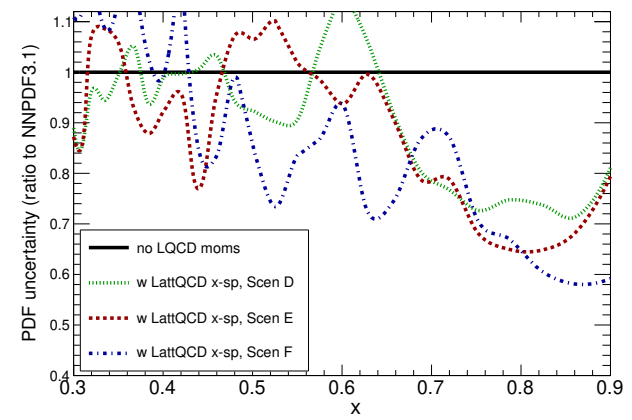

$\delta(\Delta \bar{u}) @ Q^{2}=4 \mathrm{GeV}^{2}$, NNPDFpol1.1

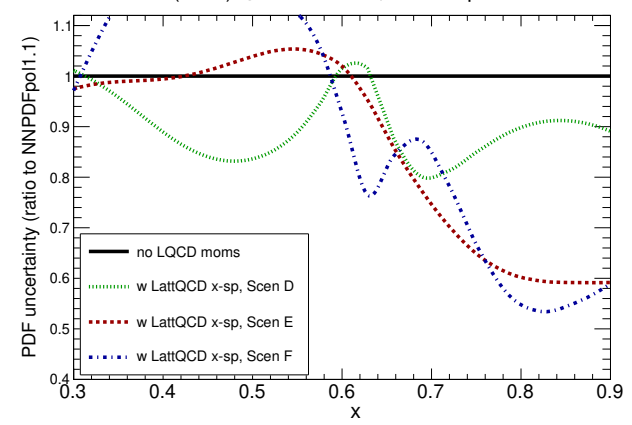

$\delta(\bar{d}) @ Q^{2}=4 \mathrm{GeV}^{2}$, NNPDF3.1

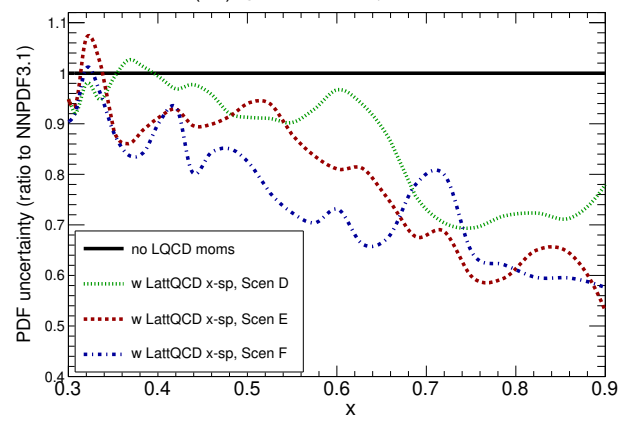

$\delta(\Delta \overline{\mathrm{d}}) @ \mathrm{Q}^{2}=4 \mathrm{GeV}^{2}$, NNPDFpol1.1

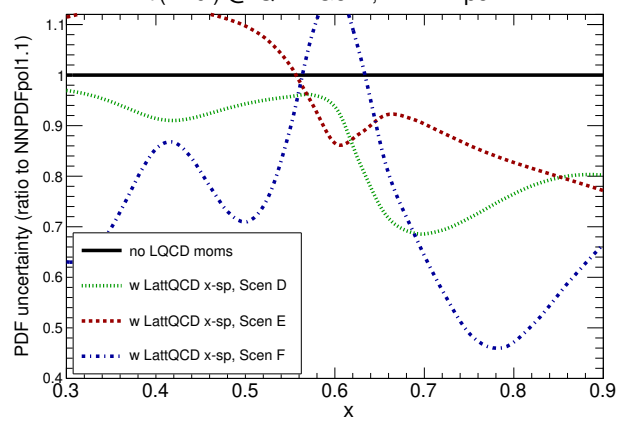

Figure 3. The ratio of PDF uncertainties to the original NNPDF3.1 (NNPDFpol1.1) in the fits where latticeQCD pseudo-data on $x$-space PDFs have been added to the global unpolarized (polarized) analysis. Specifically, we show the impact on the PDF uncertainties in $\bar{u}$ and $\bar{d}$ at large- $x$ in the upper plots, with the corresponding comparison for $\Delta \bar{u}$ and $\Delta \bar{d}$ in the lower plots.

estimates in the near future: 1) The isovector PDF at large $x$ can be used as a constraint in global PDF analyses. The large- $x$ experimental data are often contaminated by nucleus effects, which are hard to cleanly remove. Many current PDF analyses rely on extrapolation in these regions. There are ongoing LHCb measurements that can potentially improve and constrain the large- $x$ PDFs in the large- $x$ region, but the precision of these data are not yet good enough to make a difference. A recent community whitepaper among lattice and global analysis practitioners [56] predicted that a calculation of the large- $x$ isovector with $10 \%$ final error can improve on the current PDF, especially in the antiquark regions where experimental inputs are even scarcer. Currently, we are able to reproduce the global PDF results; the next step will be to plan improved calculations with total uncertainty less than $10 \%$.

2) With the promising results shown here, we can proceed with similar analyses for the less known polarized PDFs, such as transversity (the transversely polarized PDFs), where the isovector PDFs needed to make impacts for global analyses are less demanding than the unpolarized ones.

\section{Acknowledgments}

We thank the MILC Collaboration for sharing the lattices used to perform this study. The LQCD calculations were performed using the multigrid algorithm [40, 41] and Chroma software suite [42]. This research used resources of the National Energy Research Scientific Computing Center, a DOE Office of Science User Facility supported by the Office of Science of the U.S. Department of Energy under 
Contract No. DE-AC02-05CH11231 through ALCC and ERCAP; facilities of the USQCD Collaboration, which are funded by the Office of Science of the U.S. Department of Energy, and supported in part by Michigan State University through computational resources provided by the Institute for Cyber-Enabled Research. HL is supported by the US National Science Foundation under grant PHY 1653405 "CAREER: Constraining Parton Distribution Functions for New-Physics Searches".

\section{References}

[1] X. Ji, J.H. Zhang, Y. Zhao, Phys. Rev. Lett. 111, 112002 (2013), 1304.6708

[2] X. Ji, Phys. Rev. Lett. 110, 262002 (2013), 1305. 1539

[3] Y. Hatta, X. Ji, Y. Zhao, Phys. Rev. D89, 085030 (2014), 1310.4263

[4] X. Ji, Sci. China Phys. Mech. Astron. 57, 1407 (2014), 1404.6680

[5] X. Ji, J.H. Zhang, Y. Zhao, Phys. Lett. B743, 180 (2015), 1409. 6329

[6] H.W. Lin, J.W. Chen, S.D. Cohen, X. Ji, Phys. Rev. D91, 054510 (2015), 1402.1462

[7] C. Alexandrou, K. Cichy, V. Drach, E. Garcia-Ramos, K. Hadjiyiannakou, K. Jansen, F. Steffens, C. Wiese, Phys. Rev. D92, 014502 (2015), 1504 . 07455

[8] J.W. Chen, S.D. Cohen, X. Ji, H.W. Lin, J.H. Zhang, Nucl. Phys. B911, 246 (2016), 1603.06664

[9] C. Alexandrou, K. Cichy, M. Constantinou, K. Hadjiyiannakou, K. Jansen, F. Steffens, C. Wiese, Phys. Rev. D96, 014513 (2017), 1610.03689

[10] H.W. Lin, J.W. Chen, T. Ishikawa, J.H. Zhang (LP3), Phys. Rev. D98, 054504 (2018), 1708.05301

[11] J.H. Zhang, J.W. Chen, X. Ji, L. Jin, H.W. Lin, Phys. Rev. D95, 094514 (2017), 1702 . 00008

[12] J.W. Chen, L. Jin, H.W. Lin, A. Schäfer, P. Sun, Y.B. Yang, J.H. Zhang, R. Zhang, Y. Zhao (2017), 1712.10025

[13] J.W. Chen, T. Ishikawa, L. Jin, H.W. Lin, Y.B. Yang, J.H. Zhang, Y. Zhao, Phys. Rev. D97, 014505 (2018), 1706.01295

[14] C. Alexandrou, K. Cichy, M. Constantinou, K. Hadjiyiannakou, K. Jansen, H. Panagopoulos, F. Steffens, Nucl. Phys. B923, 394 (2017), 1706.00265

[15] M. Constantinou, H. Panagopoulos, Phys. Rev. D96, 054506 (2017), 1705.11193

[16] J. Green, K. Jansen, F. Steffens, Phys. Rev. Lett. 121, 022004 (2018), 1707.07152

[17] J.W. Chen, T. Ishikawa, L. Jin, H.W. Lin, Y.B. Yang, J.H. Zhang, Y. Zhao (2017), 1710.01089

[18] J.W. Chen, T. Ishikawa, L. Jin, H.W. Lin, A. Schäfer, Y.B. Yang, J.H. Zhang, Y. Zhao (2017), 1711.07858

[19] Y.Q. Ma, J.W. Qiu, Phys. Rev. D98, 074021 (2018), 1404.6860

[20] Y.Q. Ma, J.W. Qiu, Phys. Rev. Lett. 120, 022003 (2018), 1709.03018

[21] A.V. Radyushkin, Phys. Rev. D96, 034025 (2017), 1705.01488

[22] K.F. Liu, S.J. Dong, Phys. Rev. Lett. 72, 1790 (1994), hep-ph/9306299

[23] J. Liang, K.F. Liu, Y.B. Yang, EPJ Web Conf. 175, 14014 (2018), 1710. 11145

[24] W. Detmold, C.J.D. Lin, Phys. Rev. D73, 014501 (2006), hep-lat/0507007

[25] V. Braun, D. Mueller, Eur. Phys. J. C55, 349 (2008), 0709. 1348

[26] G.S. Bali et al., Eur. Phys. J. C78, 217 (2018), 1709.04325

[27] A.J. Chambers, R. Horsley, Y. Nakamura, H. Perlt, P.E.L. Rakow, G. Schierholz, A. Schiller, K. Somfleth, R.D. Young, J.M. Zanotti, Phys. Rev. Lett. 118, 242001 (2017), 1703.01153

[28] X. Xiong, X. Ji, J.H. Zhang, Y. Zhao, Phys. Rev. D90, 014051 (2014), 1310.7471

[29] A. Radyushkin, Phys. Lett. B767, 314 (2017), 1612 . 05170 
[30] K. Orginos, A. Radyushkin, J. Karpie, S. Zafeiropoulos, Phys. Rev. D96, 094503 (2017), 1706.05373

[31] H.W. Lin, Int. J. Mod. Phys. Conf. Ser. 25, 1460039 (2014)

[32] H.W. Lin, PoS LATTICE2013, 293 (2014)

[33] E. Follana, Q. Mason, C. Davies, K. Hornbostel, G.P. Lepage, J. Shigemitsu, H. Trottier, K. Wong (HPQCD, UKQCD), Phys. Rev. D75, 054502 (2007), hep-lat/0610092

[34] A. Bazavov et al. (MILC), Phys. Rev. D87, 054505 (2013), 1212 . 4768

[35] A. Hasenfratz, F. Knechtli, Phys. Rev. D64, 034504 (2001), hep-lat/0103029

[36] R. Gupta, Y.C. Jang, H.W. Lin, B. Yoon, T. Bhattacharya, Phys. Rev. D96, 114503 (2017), 1705.06834

[37] T. Bhattacharya, V. Cirigliano, S. Cohen, R. Gupta, A. Joseph, H.W. Lin, B. Yoon (PNDME), Phys. Rev. D92, 094511 (2015), 1506.06411

[38] T. Bhattacharya, V. Cirigliano, R. Gupta, H.W. Lin, B. Yoon, Phys. Rev. Lett. 115, 212002 (2015), 1506.04196

[39] T. Bhattacharya, S.D. Cohen, R. Gupta, A. Joseph, H.W. Lin, B. Yoon, Phys. Rev. D89, 094502 (2014), 1306.5435

[40] R. Babich, J. Brannick, R.C. Brower, M.A. Clark, T.A. Manteuffel, S.F. McCormick, J.C. Osborn, C. Rebbi, Phys. Rev. Lett. 105, 201602 (2010), 1005.3043

[41] J.C. Osborn, R. Babich, J. Brannick, R.C. Brower, M.A. Clark, S.D. Cohen, C. Rebbi, PoS LATTICE2010, 037 (2010), 1011.2775

[42] R.G. Edwards, B. Joo (SciDAC, LHPC, UKQCD), Nucl. Phys. Proc. Suppl. 140, 832 (2005), [,832(2004)], hep-lat/0409003

[43] G.S. Bali, B. Lang, B.U. Musch, A. Schäfer, Phys. Rev. D93, 094515 (2016), 1602.05525

[44] J.W. Chen, L. Jin, H.W. Lin, Y.S. Liu, Y.B. Yang, J.H. Zhang, Y. Zhao (2018), 1803.04393

[45] H.W. Lin, J.W. Chen, L. Jin, Y.S. Liu, Y.B. Yang, J.H. Zhang, Y. Zhao (2018), 1807.07431

[46] Y.S. Liu, J.W. Chen, L. Jin, R. Li, H.W. Lin, Y.B. Yang, J.H. Zhang, Y. Zhao (2018), 1810.05043

[47] Y.S. Liu, J.W. Chen, L. Jin, H.W. Lin, Y.B. Yang, J.H. Zhang, Y. Zhao (2018), 1807.06566

[48] S. Dulat, T.J. Hou, J. Gao, M. Guzzi, J. Huston, P. Nadolsky, J. Pumplin, C. Schmidt, D. Stump, C.P. Yuan, Phys. Rev. D93, 033006 (2016), 1506.07443

[49] R.D. Ball et al. (NNPDF), Eur. Phys. J. C77, 663 (2017), 1706.00428

[50] A. Accardi, L.T. Brady, W. Melnitchouk, J.F. Owens, N. Sato, Phys. Rev. D93, 114017 (2016), 1602.03154

[51] E.R. Nocera, R.D. Ball, S. Forte, G. Ridolfi, J. Rojo (NNPDF), Nucl. Phys. B887, 276 (2014), 1406.5539

[52] J.J. Ethier, N. Sato, W. Melnitchouk, Phys. Rev. Lett. 119, 132001 (2017), 1705.05889

[53] H.W. Lin, W. Melnitchouk, A. Prokudin, N. Sato, H. Shows, Phys. Rev. Lett. 120, 152502 (2018), 1710.09858

[54] R. Gupta, Y.C. Jang, B. Yoon, H.W. Lin, V. Cirigliano, T. Bhattacharya, Phys. Rev. D98, 034503 (2018), 1806.09006

[55] C. Alexandrou, K. Cichy, M. Constantinou, K. Jansen, A. Scapellato, F. Steffens (2018), 1807.00232

[56] H.W. Lin et al., Prog. Part. Nucl. Phys. 100, 107 (2018), 1711.07916 